\title{
Understanding the Oxidation Behavior of $\mathrm{Fe} / \mathrm{Ni} / \mathrm{Cr}$ and $\mathrm{Fe} / \mathrm{Cr} / \mathrm{Ni}$ Core/Alloy Nanoparticles
}

\author{
Laxmikant Pathade, Tennyson L. Doane, Rahiem Davon Slaton, Mathew M. Maye* \\ Department of Chemistry, Syracuse University, Syracuse, New York, 13244 \\ *mmmaye@syr.edu
}

\section{SUPPORTING INFORMATION}

\section{THEORETICAL ASSESSMENT}

Values related to thin film oxidation for $\mathrm{Fe}, \mathrm{Cr}$, and $\mathrm{Ni}$ (Table $\mathrm{S} 1$ ) and for the bulk diffusion couples shown in Figure 6 (Table S2-Table S6) were taken from the literature. Note that in compiling values from multiple references for the diffusion data we have been mindful of not reporting the same data twice, as sometimes values for the prefactor $\left(D_{0}\right)$ and the activation energy $\left(E_{a}\right)$ were within very close agreement but with slight differences in temperature or one of the variables.

The validity of the application of CM to the oxidation of metallic NPs can be further explored by considering the limiting oxide thickness experimentally observed in previous studies. Reported limiting oxide layer thicknesses for $\mathrm{Fe}, \mathrm{Ni}$, and $\mathrm{Cr}$ nano-interfaces have been reported to be $2-3 \mathrm{~nm},{ }^{1-3} 1-3 \mathrm{~nm},{ }^{4-6}$ and $\sim 3 \mathrm{~nm},{ }^{7}$ respectively. Both $\mathrm{Cr}$ and $\mathrm{Ni}$ approach this limiting rate at $3 \mathrm{~nm}$, but Fe does approach a limiting thickness implying that the values of $W, N$, and $V_{\mathrm{M}}$ need re-assessment. Interestingly, all of these $W^{\prime} \mathrm{s}$ are significantly less than those calculated from diffusion studies of bulk materials; for example, an average $W$ for Fe selfdiffusion from a compilation of bulk experiments was found to be $2.54 \mathrm{eV}$, while Fehlner's fitting of thin film oxidation found a value of $0.8 \mathrm{eV} .{ }^{8}$ Encouragingly, other researchers looking 
at the oxidation of $\mathrm{Fe}_{3} \mathrm{O}_{4} \mathrm{NPs}$ to the corresponding $\mathrm{Fe}_{2} \mathrm{O}_{3}$ products obtained $W$ in the range of $0.85-0.91 \mathrm{eV},{ }^{9,10}$ implying the use of values lower than bulk measurements is appropriate. Using $3 \mathrm{~nm}$ as the limiting thickness when the oxidation rate reaches $10^{-5} \AA / \mathrm{s}$, one can simulate the range of reasonable values for $W$ with varying $V_{M}$ (Fig S6): taking Fe as our example, if we use $V_{M}=1.6 \mathrm{~V}$, the corresponding range of activation energies would be $1.2-1.6 \mathrm{eV}$. However, if we fix $W=0.8 \mathrm{eV}$, as Fehlner did, the corresponding $V_{M}$ would be $<0.1 \mathrm{~V}$, which is exceedingly small and difficult to reconcile with the high potentials in the $\mathrm{Cr}$ and $\mathrm{Ni}$ system. It is likely, then, that in our approximation the activation energy for $\mathrm{Fe}^{2+}$ transport is larger than the $0.8 \mathrm{eV}$ value. 

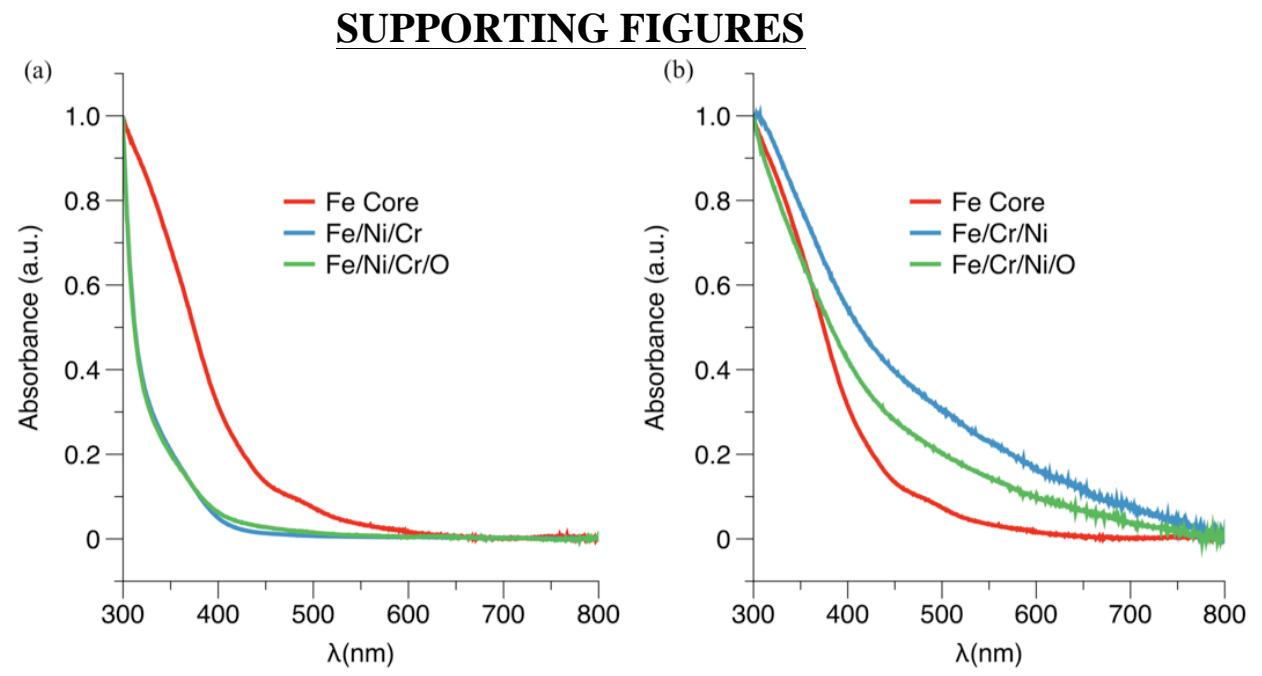

Figure S1. UV-Vis spectra for $\mathrm{Fe} / \mathrm{Ni} / \mathrm{Cr}$ (a) and $\mathrm{Fe} / \mathrm{Cr} / \mathrm{Ni}$ (b) are shown. Spectra are normalized at the $\lambda_{\mathrm{abs}(\max ) \text {. }}$


Figure S2. TGA data for $\mathrm{Fe} / \mathrm{Ni} / \mathrm{Cr}$ (a) and $\mathrm{Fe} / \mathrm{Cr} / \mathrm{Ni}$ (b) $\mathrm{NPs}$, before (red), and after (green) the intentional oxidation step. Scans were run from $100{ }^{\circ} \mathrm{C}$ to $800{ }^{\circ} \mathrm{C}$ at the rate of $10{ }^{\circ} \mathrm{C} / \mathrm{min}$ under $\mathrm{Ar} / \mathrm{O}_{2}$ atmosphere. Fe/Ni/Cr NPs lost $30.97 \%$ \& $15.36 \%$ of their total mass for unoxidized and oxidized samples, respectively. Similarly for $\mathrm{Fe} / \mathrm{Cr} / \mathrm{Ni}$ samples, mass losses were $46.57 \%$ \& $37.22 \%$. TGA mass loss values are dependent on the ligand cleanup process - which being qualitative in nature - may cause minor differences in mass loss values. 


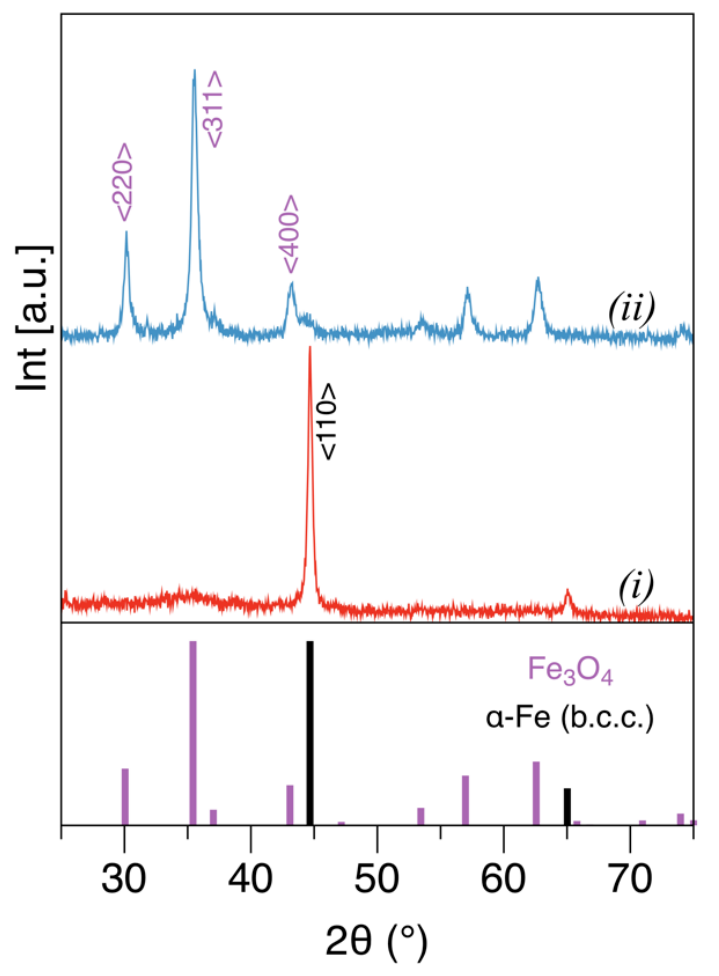

Figure S3. Representative XRD patterns for unoxidized $\alpha$-Fe cores (i), which after the oxidation step formed $\mathrm{Fe}_{3} \mathrm{O}_{4}$ (ii). 

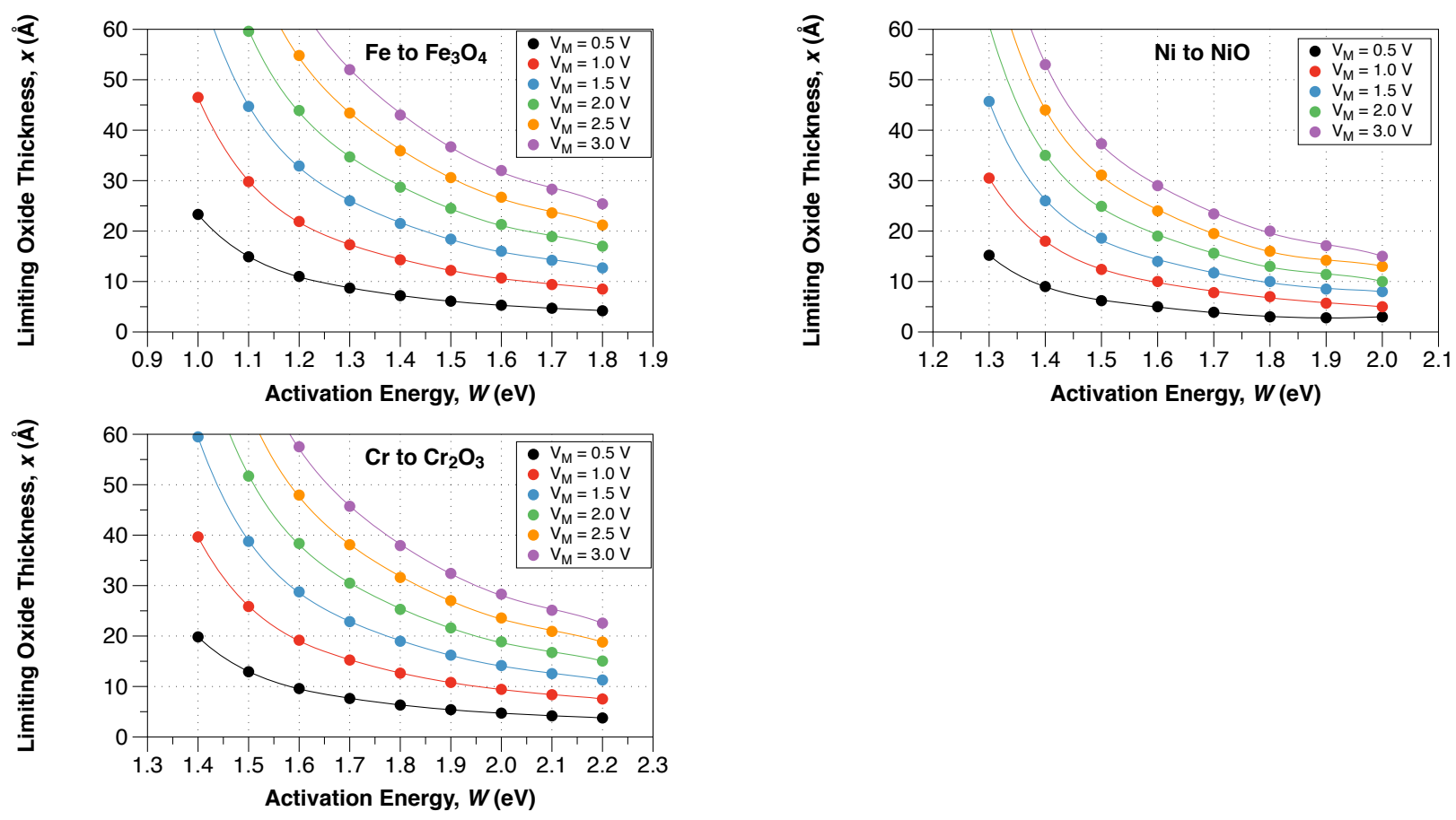

Figure S4. Simulations of the limiting thickness $x$ (where $\mathrm{dx} / \mathrm{dt}=10^{-5} \AA / \mathrm{s}$ ) for the three metals studied with variations in the activation energy of diffusion $(W)$ and the Mott potential $\left(V_{M}\right)$. Typical limiting thicknesses are in the range of 2-3 $\mathrm{nm}$.
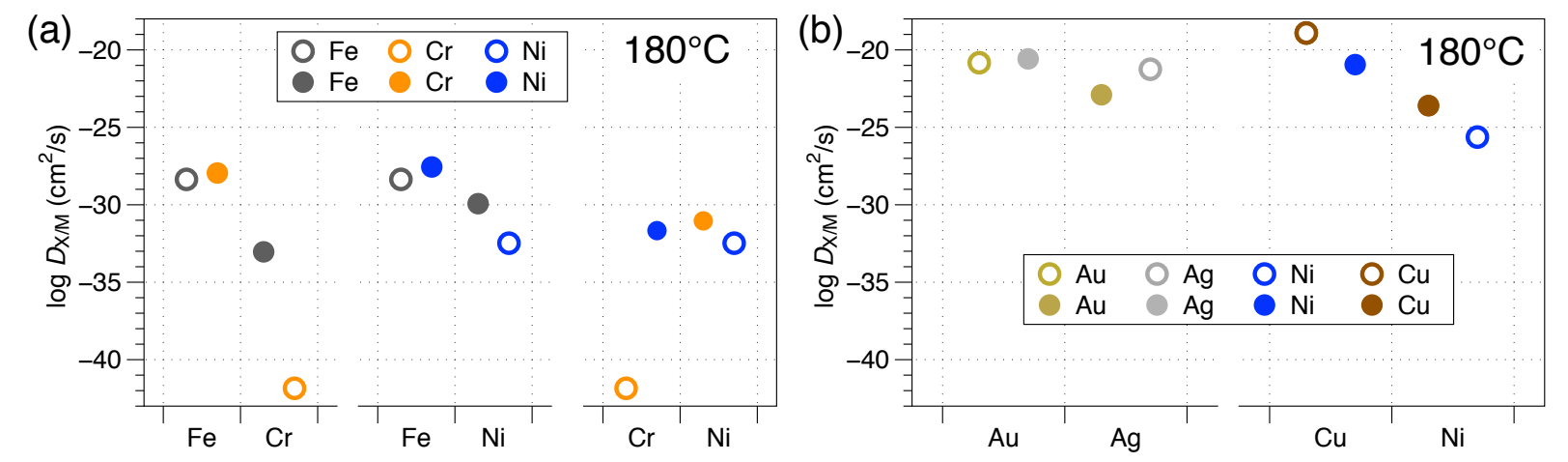

Figure S5. Comparison of the average intermetallic diffusion coefficient for the $\mathrm{Fe}-\mathrm{Cr}, \mathrm{Fe}-\mathrm{Ni}$, and $\mathrm{Cr}-\mathrm{Ni}$ couples (a) to those of $\mathrm{Au}-\mathrm{Ag}$ and $\mathrm{Ni}-\mathrm{Cu}$ (b) extrapolated to $180^{\circ} \mathrm{C}$ based on bulk measurements. Circles indicate self-diffusion while solid points depict the diffusion of a metal in the given metallic solvent indicated on the x-axis. Values for the reference diffusion couples shown in (b) were taken from reference ${ }^{11}$. 


\section{SUPPORTING TABLES}

Table S1: Values Used to Calculate Oxidation Rate as a Function of Thickness According to Fehlner $^{8}$ with a constant Mott Potential of $2.4 \mathrm{~V}$ or Oxide Induced Work Function Changes. ${ }^{12}$

\begin{tabular}{|c|c|c|c|c|c|}
\hline & & & $\mathrm{Fe}$ & $\mathrm{Ni}$ & $\mathrm{Cr}$ \\
\hline Metal Oxide & & & $\mathrm{Fe}_{3} \mathrm{O}_{4}$ & $\mathrm{NiO}$ & $\mathrm{Cr}_{2} \mathrm{O}_{3}$ \\
\hline Crystal Structure & & & Cubic & Cubic & Trigonal \\
\hline Lattice Constant (Jump Distance) & $2 a$ & $\AA$ & 8.397 & 4.2 & 4.95 \\
\hline Additional Lattice Parameter & $c$ & & - & - & 13.665 \\
\hline 1/2 Jump Distance & $a$ & $\AA$ & 4.20 & 2.10 & 2.48 \\
\hline Defect Concentration Area & $N$ & $\mathrm{~cm}^{2}$ & $5.00 \mathrm{E}+08$ & $3.00 \mathrm{E}+13$ & $5.00 \mathrm{E}+13$ \\
\hline Defect Concentration Area & $N$ & $\AA^{-2}$ & $5.00 \mathrm{E}-08$ & $3.00 \mathrm{E}-03$ & $5.00 \mathrm{E}-03$ \\
\hline Defect Activation Energy & $W$ & $\mathrm{eV}$ & 0.8 & 1.6 & 1.8 \\
\hline Charge of Migrating Species & $Z$ & & 2 & 2 & 3 \\
\hline Vibrational Frequency & $v$ & s-1 & $1.00 \mathrm{E}+12$ & $1.00 \mathrm{E}+12$ & $1.00 \mathrm{E}+12$ \\
\hline Mott Potential & $V_{M}$ & $\mathrm{~V}$ & 1.6 & 1 & 2.1 \\
\hline Metal Ions per Unit Cell & & & 24 & 4 & 12 \\
\hline Oxide Volume / Metal Ion & $\Omega$ & $\AA^{3}$ & 24.7 & 18.5 & 24.2 \\
\hline Boltzmann Constant & $K$ & $\mathrm{eV} / \mathrm{K}$ & $8.62 \mathrm{E}-05$ & 8.62E-05 & $8.62 \mathrm{E}-05$ \\
\hline Temperature & $T$ & $\mathrm{~K}$ & 373.00 & 373.00 & 373.00 \\
\hline
\end{tabular}

Table S2: Diffusion of $X$ in $\alpha-F e$

\begin{tabular}{lllllll}
\hline & $\begin{array}{l}T \\
(\mathrm{~K})\end{array}$ & $\begin{array}{l}D_{0} \\
\mathrm{~cm}^{2} / \mathrm{s}\end{array}$ & $\begin{array}{l}E_{a} \\
(\mathrm{~kJ} / \mathrm{mol})\end{array}$ & $(\mathrm{eV})$ & $\begin{array}{l}D\left(100^{\circ} \mathrm{C}\right) \\
\mathrm{cm}^{2} / \mathrm{s}\end{array}$ & Reference \\
\hline $\mathrm{Fe}$ & $973-1063$ & 2.00 & 251.2 & 2.60 & -34.87 & 11,13 \\
$\mathrm{Fe}$ & $1063-1173$ & 1.90 & 239.4 & 2.48 & -33.24 & 13 \\
$\mathrm{Fe}$ & $1133-1173$ & 2.00 & 239.9 & 2.49 & -33.28 & 13 \\
$\mathrm{Fe}$ & $911-1041$ & 27.50 & 254.1 & 2.63 & -34.13 & 13 \\
$\mathrm{Fe}$ & $1081-1157$ & 2.01 & 240.7 & 2.49 & -33.39 & 13 \\
$\mathrm{Fe}$ & $1082-1162$ & 5.40 & - & 2.62 & -34.59 & 11 \\
$\mathrm{Fe}$ & $784-1017$ & 1.02 & - & 2.53 & -34.16 & 11 \\
$\mathrm{Fe}$ & $1067-1169$ & 121.00 & - & 2.92 & -37.32 & 11 \\
$\mathrm{Fe}$ & $1052-1148$ & 2.76 & - & 2.60 & -34.62 & 11 \\
$\mathrm{Fe}$ & $1054-1163$ & 0.66 & - & 2.47 & -33.54 & 11 \\
$\mathrm{Fe}$ & $923-1123$ & 530.00 & 280.8 & 2.91 & -36.58 & 11 \\
$\mathrm{Fe}$ & $978-1173$ & 3.20 & 236.4 & 2.45 & -32.59 & 11 \\
$\mathrm{Fe}$ & $979-1168$ & 118.00 & 281.2 & 2.91 & -37.29 & 11 \\
$\mathrm{Fe}$ & $1136-1172$ & 2.00 & 239.7 & 2.48 & -33.26 & 11 \\
$\mathrm{Fe}$ & $1016-1159$ & 119.00 & 248.1 & 2.57 & -32.66 & 11
\end{tabular}




\begin{tabular}{|c|c|c|c|c|c|c|}
\hline $\mathrm{Fe}$ & $1048-1158$ & 18.00 & 268.2 & 2.78 & -36.29 & 11 \\
\hline $\mathrm{Fe}$ & - & 27.50 & 253.1 & 2.62 & -34.00 & 11 \\
\hline $\mathrm{Fe}$ & $973-1173$ & 12.00 & 277.4 & 2.88 & -37.76 & 11 \\
\hline \multirow[t]{3}{*}{$\mathrm{Fe}$} & $1082-1162$ & 5.40 & 252.3 & 2.61 & -34.59 & 14 \\
\hline & & \multicolumn{2}{|c|}{ Average } & 2.63 & -34.64 & \\
\hline & & \multicolumn{2}{|c|}{ Standard Deviation } & 0.16 & 1.63 & \\
\hline $\mathrm{Cr}$ & $1070-1150$ & 8.52 & - & 2.60 & -34.16 & 11 \\
\hline $\mathrm{Cr}$ & $775-875$ & 2.53 & 240.7 & 2.49 & -33.29 & $11,13,14$ \\
\hline $\mathrm{Cr}$ & $1043-1183$ & 2.20 & - & 2.48 & -33.20 & 11 \\
\hline \multirow[t]{3}{*}{$\mathrm{Cr}$} & 1049-1174 & 37.30 & - & 2.77 & -35.83 & 11 \\
\hline & & \multicolumn{2}{|c|}{ Average } & 2.59 & -34.12 & \\
\hline & & \multicolumn{2}{|c|}{ Standard Deviation } & 0.13 & 1.22 & \\
\hline $\mathrm{Ni}$ & $1053-1173$ & 1.30 & - & 2.43 & -32.68 & 11 \\
\hline $\mathrm{Ni}$ & $973-1173$ & 9.90 & 259.2 & 2.69 & -35.29 & 11,13 \\
\hline \multirow[t]{3}{*}{$\mathrm{Ni}$} & $953-1073$ & 1.30 & 234.3 & 2.43 & -32.69 & 14 \\
\hline & & \multicolumn{2}{|c|}{ Average } & 2.51 & -33.55 & \\
\hline & & \multicolumn{2}{|c|}{ Standard Deviation } & 0.15 & 1.51 & \\
\hline
\end{tabular}

Table S3: Diffusion of $\mathrm{X}$ in $\mathrm{Cr}$

\begin{tabular}{|c|c|c|c|c|c|c|}
\hline$X$ & $\begin{array}{l}T \\
(\mathrm{~K})\end{array}$ & $\begin{array}{l}D_{0} \\
\mathrm{~cm}^{2} / \mathrm{s} \\
\end{array}$ & $\begin{array}{l}E_{a} \\
(\mathrm{~kJ} / \mathrm{mol})\end{array}$ & $(\mathrm{eV})$ & $\begin{array}{l}D\left(100^{\circ} \mathrm{C}\right) \\
\mathrm{cm}^{2} / \mathrm{s}\end{array}$ & Reference \\
\hline $\mathrm{Cr}^{*}$ & $1473-1873$ & 0.28 & 306.5 & 3.18 & -43.46 & 13 \\
\hline $\mathrm{Cr}^{*}$ & $1303-1818$ & 0.20 & 308.6 & 3.20 & -43.90 & 13,14 \\
\hline $\mathrm{Cr}^{*}$ & $1273-2023$ & 1.60 & 339.1 & 3.51 & -47.27 & 13 \\
\hline $\mathrm{Cr}$ & $1369-2093$ & 970.00 & 435.4 & 4.51 & -57.93 & 11 \\
\hline \multirow[t]{3}{*}{$\mathrm{Cr}$} & $1073-1446$ & 46.00 & 405.5 & 4.20 & -55.07 & 11 \\
\hline & & \multicolumn{2}{|c|}{ Average } & 3.72 & -49.53 & \\
\hline & & \multicolumn{2}{|c|}{ Standard Deviation } & 0.60 & 6.61 & \\
\hline $\mathrm{Fe}$ & $1523-1700$ & 0.47 & 332.0 & 3.44 & -46.81 & 11,13 \\
\hline \multirow[t]{3}{*}{$\mathrm{Fe}$} & $1203-1513$ & 0.20 & 232.4 & 2.41 & -33.23 & 13 \\
\hline & & \multicolumn{2}{|c|}{ Average } & 2.92 & -40.02 & \\
\hline & & \multicolumn{2}{|c|}{ Standard Deviation } & 0.73 & 9.60 & \\
\hline $\mathrm{Ni}$ & 1500-1689 & 3.35 & 279.3 & 2.89 & -38.58 & 13 \\
\hline
\end{tabular}

*Nemann and Tujin (14) note that the lower values for $E_{a}$ likely reflect grain boundary diffusion, and recommend that the higher values be used for bulk diffusion. 
Table S4: Diffusion of $\mathrm{X}$ in Ni

\begin{tabular}{|c|c|c|c|c|c|c|}
\hline$X$ & $\begin{array}{l}T \\
(\mathrm{~K})\end{array}$ & $\begin{array}{l}D_{0} \\
\mathrm{~cm}^{2} / \mathrm{s}\end{array}$ & $\begin{array}{l}E_{a} \\
(\mathrm{~kJ} / \mathrm{mol})\end{array}$ & $(\mathrm{eV})$ & $\begin{array}{l}D\left(100^{\circ} \mathrm{C}\right) \\
\mathrm{cm}^{2} / \mathrm{s}\end{array}$ & Reference \\
\hline $\mathrm{Ni}$ & $1358-1573$ & 2.59 & 291.00 & 3.02 & -40.33 & 13 \\
\hline $\mathrm{Ni}$ & $1223-1523$ & 2.40 & 280.50 & 2.91 & -38.89 & 13 \\
\hline $\mathrm{Ni}$ & $748-923$ & 1.90 & 279.70 & 2.90 & -38.88 & 13 \\
\hline $\mathrm{Ni}$ & $948-1023$ & 1.90 & 279.70 & 2.90 & -38.88 & 13 \\
\hline $\mathrm{Ni}$ & $1151-1521$ & 1.27 & 279.70 & 2.90 & -39.03 & 11 \\
\hline $\mathrm{Ni}$ & $1373-1448$ & 0.40 & 267.10 & 2.77 & -37.77 & 11 \\
\hline $\mathrm{Ni}$ & $1425-1673$ & 3.36 & 292.30 & 3.03 & -40.36 & 11 \\
\hline $\mathrm{Ni}$ & $1425-1673$ & 5.12 & 297.30 & 3.08 & -40.88 & 11 \\
\hline $\mathrm{Ni}$ & $973-1373$ & 1.70 & 285.10 & 2.95 & -39.66 & 11 \\
\hline $\mathrm{Ni}$ & $1358-1576$ & 2.59 & 291.00 & 3.01 & -40.30 & 11 \\
\hline $\mathrm{Ni}$ & $1315-1667$ & 1.90 & 284.70 & 2.95 & -39.55 & 11,14 \\
\hline $\mathrm{Ni}$ & $1253-1670$ & 1.77 & 285.30 & 2.96 & -39.67 & 11 \\
\hline $\mathrm{Ni}$ & $815-1195$ & 1.33 & 281.00 & 2.91 & -39.18 & 11 \\
\hline $\mathrm{Ni}$ & $1326-1673$ & 1.82 & 285.20 & 2.95 & -39.64 & 11 \\
\hline $\mathrm{Ni}$ & $879-1670$ & 0.85 & 277.10 & 2.87 & -38.84 & 11 \\
\hline \multirow[t]{3}{*}{$\mathrm{Ni}$} & $1173-1473$ & 2.22 & 289.70 & 3.00 & -40.21 & 13 \\
\hline & & \multicolumn{2}{|c|}{ Average } & 2.94 & -39.50 & \\
\hline & & \multicolumn{2}{|c|}{ Standard Deviation } & 0.08 & 0.79 & \\
\hline $\mathrm{Fe}$ & 1409-1629 & 0.22 & 252.90 & 2.62 & -36.03 & 11 \\
\hline $\mathrm{Fe}$ & $1213-1373$ & 0.80 & 255.40 & 2.65 & -35.85 & 13 \\
\hline $\mathrm{Fe}$ & $1478-1668$ & 1.00 & 268.80 & 2.79 & -37.63 & 11,13 \\
\hline \multirow[t]{3}{*}{$\mathrm{Fe}$} & 1293-1536 & 0.074 & 245.18 & 2.54 & -35.46 & 14 \\
\hline & & \multicolumn{2}{|c|}{ Average } & 2.65 & -36.24 & \\
\hline & & \multicolumn{2}{|c|}{ Standard Deviation } & 0.10 & 0.96 & \\
\hline $\mathrm{Cr}$ & $1223-1423$ & 8.52 & 292.10 & 3.03 & -39.93 & 11 \\
\hline $\mathrm{Cr}$ & $1373-1541$ & 1.10 & 272.60 & 2.82 & -38.09 & 11,14 \\
\hline \multirow[t]{3}{*}{$\mathrm{Cr}$} & $1323-1648$ & 5.20 & 289.00 & 2.68 & -35.43 & 11 \\
\hline & & \multicolumn{2}{|c|}{ Average } & 2.84 & -37.82 & \\
\hline & & \multicolumn{2}{|c|}{ Standard Deviation } & 0.18 & 2.26 & \\
\hline
\end{tabular}




\begin{tabular}{|c|c|c|c|c|c|c|}
\hline$X$ & $\begin{array}{l}T \\
(\mathrm{~K})\end{array}$ & $\begin{array}{l}D_{0} \\
\mathrm{~cm}^{2} / \mathrm{s}\end{array}$ & $\begin{array}{l}E_{a} \\
(\mathrm{~kJ} / \mathrm{mol})\end{array}$ & $(\mathrm{eV})$ & $\begin{array}{l}D\left(100^{\circ} \mathrm{C}\right) \\
\mathrm{cm}^{2} / \mathrm{s}\end{array}$ & Reference \\
\hline $\mathrm{Cr}$ & $1423-1773$ & 4000 & 400.0 & 4.15 & -52.40 & 15 \\
\hline $\mathrm{Cr}$ & $1323-1823$ & 0.17 & 244.0 & 2.53 & -34.94 & 15 \\
\hline $\mathrm{Cr}$ & $1473-1723$ & $5.84 \mathrm{E}-09$ & 280.0 & 2.90 & -47.43 & 16 \\
\hline $\mathrm{Cr}$ & $1533-1673$ & $4.80 \mathrm{E}-04$ & 468.2 & 4.85 & -68.87 & 17 \\
\hline $\mathrm{Cr}$ & $1273-1623$ & 4000 & 419.0 & 4.34 & -55.06 & 13 \\
\hline $\mathrm{Cr}$ & $1173-1373$ & 4.29E-08 & 92.1 & 0.95 & -20.26 & 13 \\
\hline \multirow[t]{3}{*}{$\mathrm{Cr}$} & $1373-1823$ & 0.14 & 255.4 & 2.65 & -36.62 & 13 \\
\hline & & Average & & 3.31 & -45.08 & \\
\hline & & Standard Deviation & & 1.29 & 15.89 & \\
\hline $\mathrm{O}$ & $1350-1675$ & 15.9 & 419.0 & 4.34 & -57.46 & 17 \\
\hline \multirow[t]{3}{*}{$\mathrm{O}$} & $1473-1773$ & 15.9 & 403.2 & 4.18 & -55.25 & 15 \\
\hline & & Average & & 4.26 & -56.35 & \\
\hline & & Standard Deviation & & 0.12 & 1.56 & \\
\hline \multirow[t]{4}{*}{$\mathrm{Fe}$} & $1173-1373$ & 4.95E-06 & 184.0 & 1.91 & -31.06 & 13,17 \\
\hline & $1013-1373$ & 4.30E-09 & 181.0 & 1.88 & -33.71 & 18 \\
\hline & & Average & & 1.89 & -32.39 & \\
\hline & & Standard Deviation & & 0.02 & 1.87 & \\
\hline $\mathrm{Ni}$ & $1173-1373$ & 0.000000147 & 210.0 & 2.18 & -36.23 & 19 \\
\hline
\end{tabular}

Table S6: Diffusion of $\mathrm{X}$ in $\mathrm{NiO}$

\begin{tabular}{lllllll}
\hline$X$ & $T$ & $\begin{array}{l}D_{0} \\
\mathrm{~cm}^{2} / \mathrm{s}\end{array}$ & $\begin{array}{l}E_{a} \\
(\mathrm{~kJ} / \mathrm{mol})\end{array}$ & $\begin{array}{l}D\left(100^{\circ} \mathrm{C}\right) \\
(\mathrm{eV})\end{array}$ & $\begin{array}{l}\text { Reference } \\
\mathrm{cm}^{2} / \mathrm{s}\end{array}$ \\
\hline $\mathrm{Ni}$ & $1273-1743$ & 0.00183 & 182.40 & 1.89 & -28.27 & 15 \\
$\mathrm{Ni}$ & $1473-1673$ & 0.00048 & 193.60 & 2.01 & -30.42 & 15 \\
$\mathrm{Ni}$ & $1273-1673$ & 0.00044 & 176.80 & 1.83 & -28.11 & 15 \\
$\mathrm{Ni}$ & $1273-1673$ & 0.0172 & 224.00 & 2.32 & -33.12 & 15 \\
$\mathrm{Ni}$ & $1453-2033$ & 0.0477 & 243.20 & 2.52 & -35.37 & 15 \\
$\mathrm{Ni}$ & $1073-1273$ & 0.065 & 204.00 & 2.11 & -29.75 & 17 \\
$\mathrm{Ni}$ & $1373-1673$ & 0.0089 & 229.00 & 2.37 & -34.11 & 17 \\
$\mathrm{Ni}$ & $1453-2033$ & 0.0477 & 254.00 & 2.63 & -36.88 & 17 \\
$\mathrm{Ni}$ & $1123-1443$ & 0.000101 & 197.00 & 2.04 & -31.57 & 17 \\
$\mathrm{Ni}$ & $1173-1273$ & 0.041 & 230.00 & 2.38 & -33.59 & 13 \\
$\mathrm{Ni}$ & $1273-1673$ & 0.00039 & 185.10 & 1.92 & -29.32 & 13 \\
$\mathrm{Ni}$ & $973-1673$ & 0.017 & 234.50 & 2.43 & -34.60 & 13
\end{tabular}




\begin{tabular}{|c|c|c|c|c|c|c|}
\hline $\mathrm{Ni}$ & $1573-1973$ & 0.000801 & 224.00 & 2.32 & -34.46 & 13 \\
\hline $\mathrm{Ni}$ & $1273-1673$ & 0.00183 & 190.90 & 1.98 & -29.46 & 13 \\
\hline $\mathrm{Ni}$ & $1473-1673$ & 0.00048 & 202.60 & 2.10 & -31.68 & 13 \\
\hline $\mathrm{Ni}$ & $1453-2033$ & 4.77 & 254.60 & 2.64 & -34.96 & 13 \\
\hline \multirow[t]{3}{*}{$\mathrm{Ni}$} & $973-1673$ & 0.015 & 242.00 & 2.51 & -35.70 & 17 \\
\hline & & \multicolumn{2}{|l|}{ Average } & 2.24 & -32.43 & \\
\hline & & \multicolumn{2}{|c|}{ Standard Deviation } & 0.26 & 2.81 & \\
\hline \multirow{4}{*}{$\begin{array}{l}\mathrm{O} \\
\mathrm{O}\end{array}$} & $1373-1773$ & $6.20 \mathrm{E}-04$ & 230.0 & 2.38 & -35.41 & \multirow{4}{*}{$\begin{array}{l}15 \\
20\end{array}$} \\
\hline & $1373-1773$ & 50 & 540.0 & 5.60 & -73.94 & \\
\hline & & Average & & 3.99 & -54.67 & \\
\hline & & \multicolumn{2}{|c|}{ Standard Deviation } & 2.27 & 27.25 & \\
\hline $\mathrm{Fe}$ & $1272-1600$ & 0.001 & 186.8 & 1.94 & -29.15 & 16 \\
\hline $\mathrm{Fe}$ & $1073-1373$ & $1.80 \mathrm{E}-03$ & 186.3 & 1.93 & -28.83 & 13 \\
\hline $\mathrm{Fe}$ & $1273-1473$ & $2.20 \mathrm{E}-03$ & 193.4 & 2.00 & -29.73 & 13,17 \\
\hline \multirow[t]{3}{*}{$\mathrm{Fe}$} & $1334-1514$ & 0.000000592 & 108.4 & 1.12 & -21.40 & 13,17 \\
\hline & & \multicolumn{2}{|l|}{ Average } & 1.75 & -27.28 & \\
\hline & & \multicolumn{2}{|c|}{ Standard Deviation } & 0.42 & 3.93 & \\
\hline $\mathrm{Cr}$ & $1603-1853$ & 0.004 & 230.0 & 2.38 & -34.60 & 13 \\
\hline $\mathrm{Cr}$ & $1373-1523$ & $9.36 \mathrm{E}-05$ & 197.0 & 2.04 & -31.61 & 13,17 \\
\hline \multirow[t]{3}{*}{$\mathrm{Cr}$} & $1465-1915$ & 0.0086 & 282.2 & 2.92 & -41.57 & 13,17 \\
\hline & & \multirow{2}{*}{\multicolumn{2}{|c|}{$\begin{array}{l}\text { Average } \\
\text { Standard Deviation }\end{array}$}} & 2.45 & -35.93 & \\
\hline & & & & 0.4 & 5.11 & \\
\hline
\end{tabular}




\section{Reference:}

(1) Peng, S.; Wang, C.; Xie, J.; Sun, S. Synthesis and Stabilization of Monodisperse Fe Nanoparticles. J. Am. Chem. Soc. 2006, 128, 10676-10677.

(2) Cabot, A.; Ibáñez, M.; Guardia, P.; Alivisatos, A. P. Reaction Regimes on the Synthesis of Hollow Particles by the Kirkendall Effect. J. Am. Chem. Soc. 2009, 131, 11326-11328.

(3) Yoon, T.-J.; Shao, H.; Weissleder, R.; Lee, H. Oxidation Kinetics and Magnetic Properties of Elemental Iron Nanoparticles. Part. Part. Syst. Charact. 2013, 30, 667-671.

(4) Johnston-Peck, A. C.; Wang, J.; Tracy, J. B. Synthesis and Structural and Magnetic Characterization of $\mathrm{Ni}$ (Core)/NiO(Shell) Nanoparticles. ACS Nano 2009, 3, 1077-1084.

(5) Railsback, J. G.; Johnston-Peck, A. C.; Wang, J.; Tracy, J. B. Size-Dependent Nanoscale Kirkendall Effect During the Oxidation of Nickel Nanoparticles. ACS Nano 2010, 4, 1913-1920.

(6) Wang, C.-M.; Genc, A.; Cheng, H.; Pullan, L.; Baer, D. R.; Bruemmer, S. M. In-Situ TEM Visualization of Vacancy Injection and Chemical Partition during Oxidation of Ni-Cr Nanoparticles. Sci. Rep. 2014, 4.

(7) Wu, W.; Maye, M. M. Void Coalescence in Core/Alloy Nanoparticles with Stainless Interfaces. Small 2014, 10, 271-276.

(8) Fehlner, F. P. Low Temperature Oxidation of Metals and Semiconductors. J. Electrochem. Soc. 1984, 131, 1645.

(9) Sidhu, P. S.; Gilkes, R. J.; Posner, A. M. Mechanism of the Low Temperature Oxidation of Synthetic Magnetites. J. Inorg. Nucl. Chem. 1977, 39, 1953-1958.

(10) Tang, J.; Myers, M.; Bosnick, K. A.; Brus, L. E. Magnetite Fe ${ }_{3} \mathrm{O}_{4}$ Nanocrystals: Spectroscopic Observation of Aqueous Oxidation Kinetics ${ }^{\dagger}$. J. Phys. Chem. B 2003, 107, 7501-7506.

(11) Neumann, G.; Tuijn, C. Self-Diffusion and Impurity Diffusion in Pure Metals: Handbook of Experimental Data; Pergamon materials series; 1st ed.; Pergamon: Amsterdam ; Boston ; London, 2009.

(12) Quinn, C. M.; Roberts, M. W. Chemisorption of Oxygen and Subsequent Processes on Metal Films : Work Function Measurements. Trans. Faraday Soc. 1964, 60, 899.

(13) Mrowec, S. Defects and Diffusion in Solids: An Introduction; Materials science monographs; Elsevier Scientific Pub. Co. ; distribution for the U.S.A. and Canada, Elsevier/North-Holland: Amsterdam ; New York : New York, 1980.

(14) CRC Materials Science and Engineering Handbook; Shackelford, J. F.; Alexander, W.; Park, J. S., Eds.; 2nd ed.; CRC Press: Boca Raton, 1994.

(15) Kofstad, P. Nonstoichiometry, Diffusion, and Electrical Conductivity in Binary Metal Oxides; Wiley series on the science and technology of materials; Wiley-Interscience: New York, 1972.

(16) Fisher, D. J. Diffusion and Ionic Conduction in Oxides; 2007.

(17) Freer, R. Self-Diffusion and Impurity Diffusion in Oxides. J. Mater. Sci. 1980, 15, 803824.

(18) Sabioni, A. C. S.; Huntz, A. M.; Silva, F.; Jomard, F. Diffusion of Iron in Cr2O3: Polycrystals and Thin Films. Mater. Sci. Eng. A 2005, 392, 254-261.

(19) Sabioni, A. C. S.; Huntz, A. M.; Souza, J. N. V.; Martins, M. D.; Jomard, F. Diffusion of Nickel in Single- and Polycrystalline $\mathrm{Cr}_{2} \mathrm{O}_{3}$. Philos. Mag. 2008, 88, 391-405.

(20) Dubois, C.; Monty, C.; Philibert, J. Influence of Oxygen Pressure on Oxygen Self-Diffusion in NiO. Solid State Ion. 1984, 12, 75-78. 